\title{
Feature
}

\section{Alternative fat sources to animal fat for pigs}

\author{
Charlotte Lauridsen, Thomas Bruun Christensen, Ulrich Halekoh, and Søren Krogh Jensen
}

Charlotte Lauridsen is Senior Scientist at Aarhus University, Research Centre Foulum, Department of Animal Health, Welfare and Nutrition, P.O. Box 50, 8830 Tjele, Denmark; tel: +45-89-991-238, fax: +45-89-991-166, e-mail: Charlotte.Lauridsen@agrsci.dk

Thomas Bruun Christensen is MSc student, Royal Veterinary and Agricultural University, Copenhagen, Denmark

Ulrich Halekoh is Senior Scientist at the Danish Institute of Agricultural Sciences, Research Centre Foulum, P.O. Box 50, 8830 Tjele, Denmark, tel: +45-89-9918-250, fax: +45-8999-1300, e-mail: ulrich.halekoh@ agrsci.dk

Søren Krogh Jensen is Senior Scientist at Aarhus University, Research Centre Foulum, Department of Animal Health, Welfare and Nutrition, P.O. Box 50, 8830 Tjele, Denmark; tel: +45 89 991-117, fax: +45-89-991-166, e-mail: SorenKrogh.Jensen @agrsci.dk

\section{Summary}

The use of fats and oils in diets for pigs is of great importance due to their high energy value. As a consequence of the BSE-crisis in the European Union, the amount of animal fat available for animal feeds has been reduced, and alternative fat sources are of increasing importance. In this paper, we review our main findings on the effects of diets with different fat sources on apparent fat digestibility in pigs. A method for quantitative measurement of fat extraction from feed and faeces has been developed, and this method has been used in a digestibility trial, in which diets added 5\% of either animal fat, palm oil mix, palm oil, vegetable oil mix, coconut oil, or rapeseed oil were tested in weaned and growing pigs. It was concluded that several vegetable fat sources (palm oil mix, palm oil, coconut oil, rapeseed oil) could be used as alternatives to animal fat in pig feed, whereas fat blends should be avoided.

\section{Introduction}

The feed cost is the most important cost in pig production and energy represents the greatest proportion of this cost. Due to their high energy value, being approximately 2.25 times that of carbohydrates, the use of fats and oils in diets for pigs is of great importance. Among the commonly used fat sources in pig feed are animal fat (lard, tallow), vegetable fat sources (coconut oil, palm oil, palm oil mix, corn oil, rapeseed oil and soybean oil) and marine fat sources (fish oil). Animal fat has largely been used in pig feed although its energy value is generally lower than the energy value of vegetable fat sources or fish oil. In spite of this, the higher content of saturated fatty acids (mainly 16:0 and 18:0) makes animal fat preferred, since high levels of unsaturated fatty acids from vegetable or fish oil may result in reduced product quality. However, due to the BSE-crisis, the use of feedstuffs of animal origin (i.e. animal fat) has been reduced under European conditions, and this has led to use of vegetable oils as a substitute for animal fat in pig diets. The chemical and physical properties (i.e. chain length, saturation, free fatty acid content and melting point) and hence the nutritional value differs widely between vegetable oils, animal fats, and by-products from the fat industry. The postweaning digestibility often varies between different dietary fat sources, and vegetable fat sources (in particular corn oil, coconut oil and soybean oil) are more digestible than animal fat sources. Although several experiments with varying levels of dietary fats have been published, information concerning different dietary fat sources for growing pigs is scarce. It is generally accepted that the apparent digestibility of fat is less affected by dietary fat source in growing pigs than in weaned piglets, however, according to the author's knowledge, no recent studies have considered the digestibility of different fat sources in pigs using the same individuals throughout the weaning and growing periods. This article is concerned with the comparison of the apparent fat digestibility of feeds made with either vegetable or animal fat using a modified Bligh and Dyer method for extraction of fat from feed and faeces.

\section{Extraction of fat}

Apparent fat digestibility is calculated as the difference between ingested fat in feed and excreted fat. Thus, in order to evaluate the digestibility of dietary fat, extraction of fat in feed and faeces is required. A broad range of extraction methods exist, and the evaluation and discussion of the specific extraction methods is complex. In our laboratory we have traditionally used the method of Stoldt (1952), which is a Soxhlet extraction with prior acid hydrolysis, and using diethyl ether or petroleum ether as common solvent, followed by a gravimetrically determination of the extracted fat content. However, the method is slow and solvents are used in considerable amounts. In the method described by Bligh and Dyer (1952), a stepwise application of chloroform, methanol and water is used to extract the lipids into the chloroform phase, and after evaporation, the fat content can be determined gravimetrically. Several modifications to the Bligh and Dyer method have been suggested, and we compared a modified Bligh and Dyer extraction, where a step with boiling $3 \mathrm{M} \mathrm{HCl}$ has been included prior to the extraction step, with that of Stoldt for the quantitative determination of total fat in feed and faeces: Prior to extraction, feed and faeces samples $(500 \mathrm{~g})$ were hydrolysed in $1.5 \mathrm{ml}$ of $3 \mathrm{M}$ hydrochloric acid and $80^{\circ} \mathrm{C}$ for $1 \mathrm{~h}$. Fat was extracted in three steps, first methanol $(3.00 \mathrm{ml})$ and chloroform $(1.50 \mathrm{ml})$ were added, and samples were shaken for $30 \mathrm{~s}$, finally water $(1.50 \mathrm{ml})$ was added and shaking was repeated. The chloroform and water-methanol phases were separated by centrifugation $(1500 \mathrm{rpm})$ for $15 \mathrm{~min}$. Three 

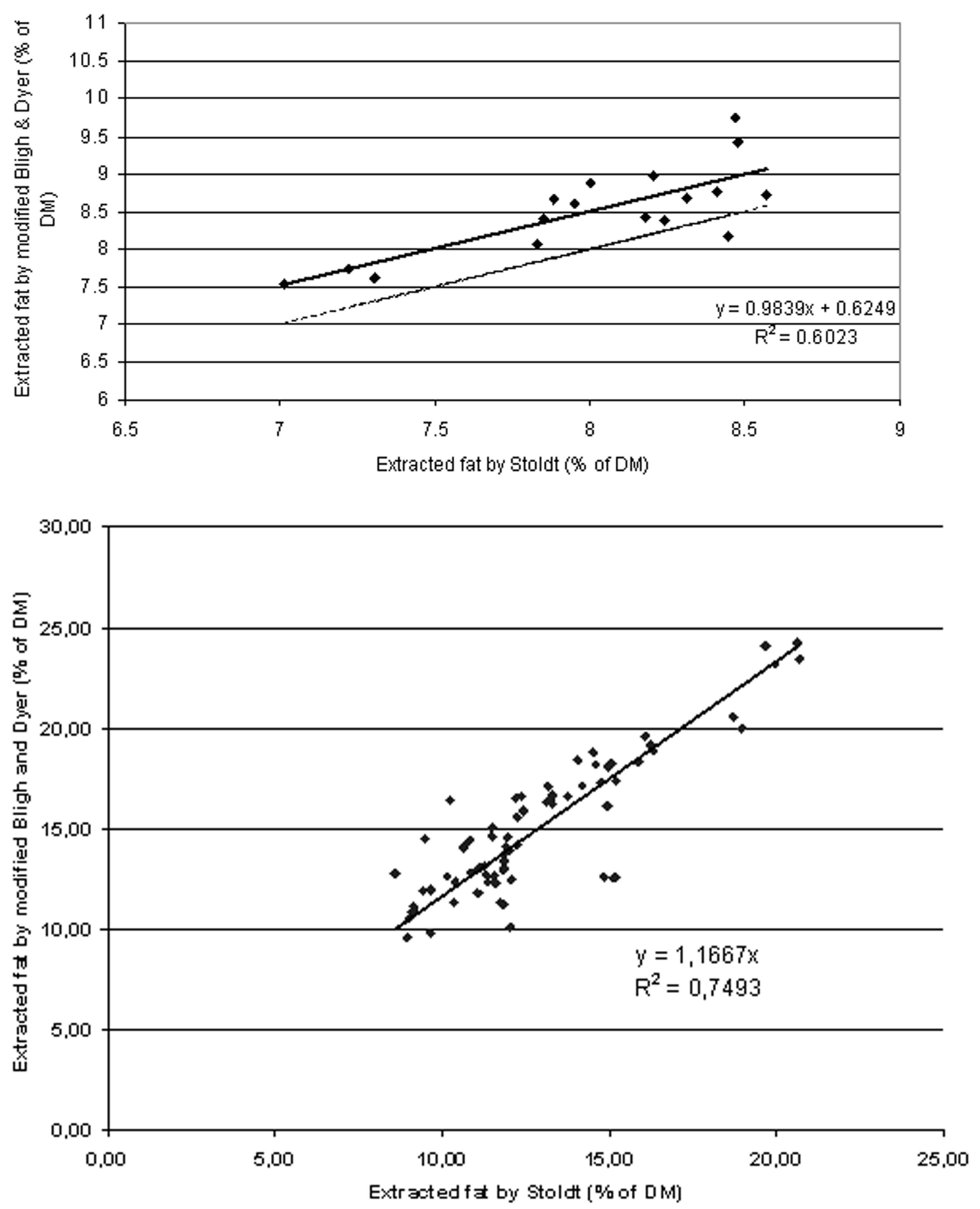

Figure 1. a) Regression line showing the correlation between extracted fat in feed samples (17 diets with dietary fat added at a $5 \%$ level) extracted with either Stoldt or modified Bligh \& Dyer. The dashed reference line shows the point of complete agreement between the two methods. b) Regression line showing the correlation between extracted fat in faeces samples (72 faeces samples of pigs fed diets with fat added at a $5 \%$ level) extracted with either Stoldt or modified Bligh \& Dyer. DM - dry matter.

$\mathrm{ml}$ of the chloroform phase was transferred to a new flask, and a quantitative determination of total fat is carried out by evaporation under constant nitrogen flux. For comparison, total fat in the diets and faeces was analysed according to Stoldt (1952) using petroleum ether as a solvent.

A shown in Fig. 1, the modified Bligh and Dyer method extracted more fat from feed than the Stoldt method when considering the total amount of extracted fat in \% of dry matter. The correlation between the two methods was not strong $\left(\mathrm{R}^{2}=\right.$ 0.602 ), but the slope of the fitted regression line is very close to 1 thus indicting that the two methods were comparable. In Fig. 2, the total amount of extracted fat in $\%$ of dry matter in faeces according to the two methods is presented. The correlation between the two methods on faeces was larger than observed for the feed $\left(R^{2}=0.742\right)$, and the Bligh and Dyer method extracted more fat from faeces than the Stoldt method.

\section{Apparent fat and energy digestibility}

The modified Bligh and Dyer method was used to study the apparent fat digestibility of feed containing $5 \%$ of different fat sources (animal fat, palm oil mix, palm oil, vegetable oil mix, coconut oil and rapeseed oil). In brief, 48 pigs of 8 litters (6 piglets per litter) were obtained from the herd at the Research Centre Foulum at weaning ( 28 days of age) and were moved to metabolism cages. After 2 days of adaptation, three consecutive balance periods each of 5 days were followed by an intervening period of 2 days between the balance periods. The experiment was resumed when the average weight of the pigs was approximately $50 \mathrm{~kg}$, and the intervening periods between the balance trials were extended to 9 days. Thus, a total of 6 balance periods were carried out, in weaned piglets on days 30-35, days 37-42, days 44-49 for balance periods $1-3$, respectively, and in growing pigs 
Table 1. Gross energy content (GE) and fatty acid composition of dietary fats and oils used in this experiment.

\begin{tabular}{|c|c|c|c|c|c|c|c|}
\hline & \multicolumn{7}{|c|}{ Fat source } \\
\hline & Animal fat & Palm oil mix & Palm oil & $\begin{array}{l}\text { Vegetable } \\
\text { oil mix }{ }^{1}\end{array}$ & Coconut oil & Rapeseed oil & Fat blend \\
\hline $\mathrm{GE}\left(\mathrm{MJ} \mathrm{kg}^{-1}\right)$ & 38.5 & 38.2 & 38.4 & 38.3 & 36.7 & 38.7 & $n d^{2}$ \\
\hline \multicolumn{8}{|l|}{ Fatty acids (g 100g-1 DM) } \\
\hline 8:0 & 0.00 & 0.00 & 0.00 & 0.00 & 6.35 & 0.00 & 0.00 \\
\hline $10: 0$ & 0.09 & 0.00 & 0.08 & 0.04 & 5.33 & 0.00 & 0.18 \\
\hline $12: 0$ & 0.10 & 0.19 & 0.18 & 0.19 & 42.5 & 0.00 & 2.10 \\
\hline $14: 0$ & 1.29 & 1.04 & 1.00 & 1.02 & 16.5 & 0.00 & 1.60 \\
\hline $16: 0$ & 24.1 & 38.2 & 42.4 & 40.3 & 8.60 & 4.60 & 36.7 \\
\hline $16: 1$ & 2.72 & 0.50 & 0.13 & 0.32 & 0.47 & 0.17 & 0.13 \\
\hline 18:0 & 13.7 & 5.82 & 4.28 & 5.05 & 2.66 & 1.59 & 5.85 \\
\hline $18: 1$ & 39.2 & 35.6 & 38.6 & 37.1 & 6.97 & 60.2 & 35.4 \\
\hline $18: 2$ & 7.88 & 9.85 & 9.94 & 9.90 & 2.00 & 19.1 & 10.1 \\
\hline $18: 3(n-3)$ & 0.62 & 0.53 & 0.23 & 0.38 & 0.00 & 9.45 & 0.00 \\
\hline $20: 0$ & 0.04 & 0.23 & 0.31 & 0.27 & 0.00 & 0.40 & 0.39 \\
\hline $20: 1$ & 0.89 & 0.29 & 0.12 & 0.21 & 0.00 & 1.10 & 0.18 \\
\hline $20: 2$ & 0.42 & 0.08 & 0.00 & 0.04 & 0.00 & 0.00 & 0.00 \\
\hline $20: 4(n-6)$ & 0.22 & 0.00 & 0.00 & 0.00 & 0.00 & 0.00 & 0.00 \\
\hline $22: 0$ & 0.00 & 0.00 & 0.00 & 0.00 & 0.00 & 0.23 & 0.09 \\
\hline$\Sigma$ Fatty acids & 91.6 & 92.3 & 97.3 & 94.8 & 91.4 & 96.9 & 92.7 \\
\hline$\Sigma$ Saturated & 39.3 & 45.5 & 48.3 & 46.9 & 81.9 & 6.8 & 46.9 \\
\hline$\Sigma$ Monounsaturated & 42.8 & 36.4 & 38.9 & 37.6 & 7.45 & 61.5 & 35.7 \\
\hline$\Sigma$ Polyunsaturated & 9.13 & 10.5 & 10.2 & 10.3 & 2.00 & 28.6 & 10.1 \\
\hline Unsaturated/saturated & 1.32 & 1.03 & 1.02 & 1.03 & 0.12 & 13.2 & 0.98 \\
\hline$\Sigma$ Medium chain fatty acid ${ }^{3}$ & 1.48 & 1.23 & 1.26 & 1.25 & 70.7 & 0.00 & 3.88 \\
\hline$\Sigma$ Long chain fatty acids ${ }^{4}$ & 89.8 & 91.1 & 96.0 & 93.6 & 20.7 & 96.7 & 88.8 \\
\hline Free fatty acids & 8.25 & 73.2 & 13.0 & 43.1 & 0.00 & 0.41 & 33.6 \\
\hline
\end{tabular}

${ }^{1}$ Calculated as the mean between palm oil mix and palm oil

2 nd, not determined.

${ }^{3} 8: 0,10: 0,12: 0,14: 0$

4 16:0-20:4

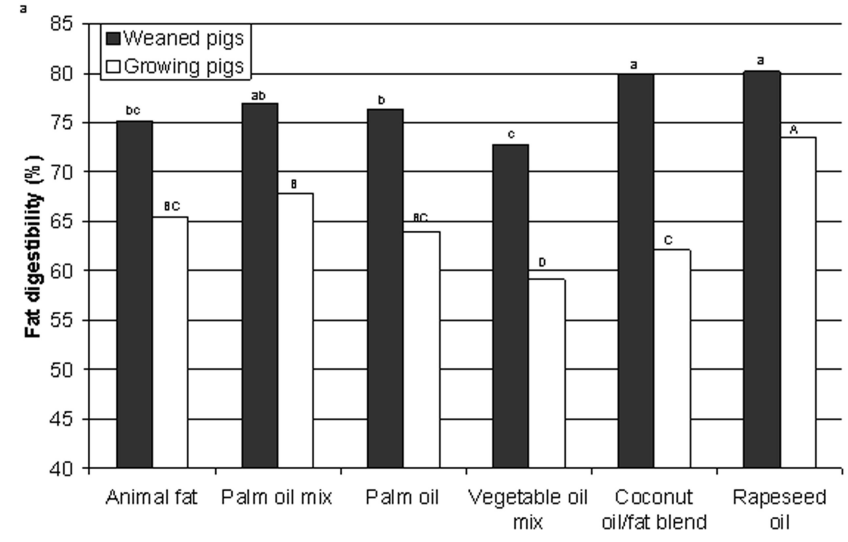

Figure 2. Effect of dietary fat sources in apparent fat digestibility of weaned and growing pigs (SE=1.1 and $1.5 \%$ for weaners and growers, respectively). Means with different letters within each group of pigs are significantly different $(P<0.05)$

balance periods 4-6 with initial weights of $51.7 \mathrm{~kg}, 67.0 \mathrm{~kg}$, and $82.1 \mathrm{~kg}$, respectively. The details of the experimental conditions are given elsewhere (Lauridsen et al., 2005). We hypothesized that the apparent digestibility of fat would differ between dietary fat sources.

The experiment used six different fat sources added at a level of 5\% 1) animal fat (of swine), 2) palm oil mix (primarily palm oil fatty acid distillates, 3) palm oil, 4) vegetable oil mix (50\% palm oil mix $+50 \%$ palm oil), 5) coconut oil and rapeseed oil. During the growing phase, coconut oil was replaced with a fat blend (65\% palm oil $+15 \%$ palm oil mix $+20 \%$ mixed fatty acids). All vegetable fat sources were obtained from DLG (Dansk Landbrugs Grovvareselskab, Copenhagen, Denmark), and animal fat from DAKA (Randers, Denmark). In Table 1, the chemical composition of the dietary fats and oils is given. Content of gross energy was almost similar between fat sources, although coconut oil had a slightly lower content. It should be noted that the content of free fatty acids varied considerably between the dietary fat sources. Because each pig received one type of dietary fat during all experimental periods, the statistical design made it possible to evaluate the combined effect of age of the pig (weaned versus growing pigs) and added dietary fat on apparent fat digestibility.

The results of the balance trial showed that in weaned piglets, rapeseed oil and coconut oil diets lead to a higher apparent fat digestibility $(80.0-80.2 \%)$ than animal fat, palm oil and vegetable oil mix diets $(72.8-76.4 \%)$. The palm oil mix diet was intermediate (77.0\%), and as indicated by the letters in Fig. 2, no statistically significant differences could be obtained with regard to the palm oil mix and other fat sources except the vegetable oil mix. The apparent fat digestibility increased from days 30-42 to days 44-49. In growing pigs, the apparent fat digestibility was lower than in weaned piglets. The rapeseed oil diet had a higher apparent fat digestibility (73.5\%) than the other diets, whereas animal fat, palm oil mix, fat blend diets were intermediate (62.1-67.8\%) and that of vegetable oil mix diet (59.1\%) was significantly lower than all other diets. No effect of dietary fat sources was observed with regard to the apparent protein digestibility of the pigs. 
This balance study was the first one comparing palm oil mix and palm oil in diets for weaned pigs, and it showed that both fat sources were potential alternatives to animal fat. Because of a similar fatty acid profile, it was expected that the apparent fat digestibility of the palm oil would be quite similar to the apparent fat digestibility obtained with the animal fat diet. However, given the high content of free fatty acids in the palm oil mix it was surprising that this diet resulted in a comparable result to animal fat and palm oil. Free fatty acids may decrease the digestibility of the fat due to a reduced capability by the pig to form mixed micelles, which is a prerequisite for lipid absorption. In spite of the fat digestibility observed for the palm oil mix, it was therefore highly unexpected that the vegetable oil mix, which was composed of $50 \%$ palm oil and $50 \%$ palm oil mix resulted in a lower apparent fat digestibility than animal fat, palm oil mix and palm oil, given the fact that this fat source had approximately half the content of free fatty acids of the palm oil mix. [In addition, when taking into consideration that the palm oil mix contained $5-10 \%$ palm oil, the present result is even more surprising]. The coconut oil diet had a higher apparent fat digestibility than animal fat probably because of its content of medium-chained fatty acids, which are more water soluble, facilitating the absorption through the unstirred water layer in comparison to long chain fatty acids. This would be even more compromised when lipase and bile secretion is insufficient, and in this case the presence of phospholipids (e.g. lecithin) may increase the apparent fat digestibility.

Previous studies (e.g. Cera et al., 1990) have shown that the apparent fat digestibility increased with age, however, no studies have so far followed the digestibility in pigs from weaning to slaughter. It is generally believed that the secretion of lipases and bile is sufficient in growing pigs, but could be limited in weaners, whereby the apparent fat digestibility in growers would be expected to be higher than in weaners. In a recent study (Lauridsen et al., 2007) we investigated the effect of dietary fat sources for sows on the capability of fat digestion (amongst others lipase activity and bile salt concentration) by the piglets while suckling and after weaning, and we found that age rather than dietary fat sources influenced the lipid absorption capacity. The activities of pancreatic enzymes (e.g. lipase) were at the lowest level in weeks 1 and 2 after weaning, whereas the concentration of bile salts increased with progressing age of the piglets.

In general, the fat digestibility observed in the present experiment was low in comparison with the results of previously published studies. However, this may be due to the fact that the modified Bligh and Dyer method was more efficient in fat extraction than the Stoldt method, which will lead to a lower apparent fat digestibility. Chloroform (as used during the fat extraction method of the present experiment) is a better solvent for polar lipids than petroleum ether (as used in the Stoldtmethod). However, another factor could be the dietary level of fat (5\%), which was lower than for other experiments investigating effects of dietary fat sources on the fat digestibility. It is wellknown that the apparent fat digestibility increases when the level of added dietary fat is increased. Since most of the previous experiments have investigated the effect of dietary fat sources on fat digestibility of either weaners or growers, and have used a higher dietary level of fat, using less efficient fat extraction methods, these factors may explain the differing results observed in our experiment.

\section{Performance}

In order to access the suitability of the fat sources for animal nutrition other factors such as palatability of feed and cost effectiveness must also be considered. In the present study, the dietary fat sources did not affect daily gain or feed conversion in either weaned piglets or growing pigs, but it should be kept in mind that the limited number of pigs per treatment would not be sufficient to make any conclusions with regard to the productivity. In addition, when pigs were performing the balance trial, they were fed semi-ad libitum, i.e., the pigs were able to empty the trough between feeding. This experimental condition is different from practise in which ad libitum feeding from weaning to slaughter is common. However, in concurrent large-scale productivity trial (Maribo, 2005) using the same diets for weaned piglets, no effect of dietary fat source in productivity was found, but based on economical calculations and using animal fat as a reference (index $=100$ ), coconut oil (index $=107$ ) and palm oil (index $=108$ ) came out with the highest production value, and rapeseed oil was the only source resulting in a significantly lower production value than animal fat. The reason for that was unclear.

\section{Conclusions}

Our data show that several vegetable fat sources (palm oil mix, palm oil, coconut oil, rapeseed oil) could be used as alternatives to animal fat in pig feed, whereas fat blends should be avoided. The apparent fat digestibility was lower in growing pigs than in weaners, which was in contrast to the existing literature. However, the fat extraction method used, which is a modification of Bligh and Dyer, was more efficient than the commonly used Stoldt method, whereby the apparent fat digestibility would be lower. In addition, the dietary fat level may influence the apparent fat digestibility.

\section{Acknowledgement}

The authors wish to thank Mette Würtz, Henrik Benzon and Susanne Marian Urban for their technical assistance within the reported experiment.

\section{References}

1. Bligh, E.G. and Dyer, W.J. (1959) Can. J. Biochem. Physiol., 37, 911-917.

2. Cera, K. R., et al. (1990) J. Anim. Sci., 68, 2756-2765.

3. Lauridsen, C., et al. (2005). In: Manipulating pig production X. Proc. Tenth Biennial Conference of the Australasian Pig Science Association (Inc.) (APSA), Paterson, J.E. (ed.), November 27-30, Christchurch, New Zealand, 274.

4. Lauridsen, C., Hedemann, M.S., et al. (2007). Livestock Sci., in press.

5. Maribo, H. (2005) Danish Pig Production, Meddelelse 719.

6. Stoldt, W. (1952) Fette, Seifen, Anstrichmittel, 54, 206-207. 